\title{
Introduction to the thematic issue on selected topics from Intelligent Environments 2017
}

\author{
Jason J. Jung a,* and Paulo Novais ${ }^{\mathrm{b}}$ \\ a Department of Computer Engineering, Chung-Ang University, Korea \\ ${ }^{\mathrm{b}}$ Departamento de Informatica, Universidade do Minho, Portugal
}

\section{Introduction}

Intelligent Environments (IEs) refer to physical spaces into which IT and other pervasive computing technologies are woven and are used to achieve specific goals for the user, the environment, or both. IEs have the ultimate objective of enriching user experience, better manage and increase user awareness of that environment.

The 13th International Conference on Intelligent Environments (IE 17) was held at Seoul, Republic of Korea, and it was a great chance to share the state of the art data analytics methods and technologies. Based on the recommendations from conference committee members, four papers have been invited for this thematic issue.

\section{In this thematic issue}

The thematic issue starts with the article " $A k$ nearest neighbors based approach applied to more realistic activity recognition datasets". It presents data mining methods for human activity recognition. Particularly, it focuses on data clustering approach (i.e., knearest neighborhood method) in order to improve the performance with real world datasets.

The article "Analysis and verification of ECA rules in Intelligent Environments" studies how to represent and process event-condition-action (ECA) rules in various environments. A system called vIRONy has been designed and implemented for considering Satisfiability Modulo Theory (SMT) and analyzing the satisfi- ability and reliability. This study claims that the proposed contribution can be applied for smart home and e-health domains.

The article "Real-time crowd management for cultural heritage events: A case study on carnival parades" presents an interesting smart application for cultural heritage sites. This mobile application (called PARAIDS) is based on real time decision making process. The application has been particularly applied to carnival parades.

The article "An unsupervised technique to discretize numeric values by fuzzy partitions" has investigated how to discretize numerical values for data mining pre-processing step. Especially, it presents an unsupervised approach on extreme learning machine classifier. It shows that the proposed approach outperforms the $\mathrm{k}$-means discretization method.

\section{Acknowledgements}

This thematic issue has been achieved by a number of fruitful collaborations. We would like to thank the Editor in Chief of Journal of Ambient Intelligence and Smart Environments (JAISE), Juan Carlos Augusto, for his kind support and help during the entire process of publication. This work was possible thanks to the work of the renowned researchers that provided their anonymous reviews. Finally, we are most grateful to the authors for their valuable contributions and for their willingness and efforts to improve their papers in accordance with the suggestions and comments from reviewers.

\footnotetext{
*Corresponding author. E-mail: j2jung@gmail.com.
} 